\title{
EDITORIAL
}

\section{The challenge of defining relative adrenal insufficiency}

\section{Journal of Perinatology (2012) 32, 397-398; doi:10.1038/jp.2012.21}

Defining relative adrenal insufficiency in preterm infants remains a significant challenge for neonatologists. The phenomenon of relative adrenal insufficiency in adults and children has been well described, with normal basal levels of cortisol, yet inadequate response to stress as documented by a blunted response to adrenocorticotropin hormone (ACTH) stimulation. ${ }^{1-3}$ In preterm infants, numerous confounding factors make the definition more elusive. Preterm infants developmentally may have low basal cortisol levels in the absence of symptomatology, ${ }^{4}$ and it is less clear what constitutes an appropriate stress response in the face of an acute illness, such as respiratory distress syndrome or sepsis. ${ }^{5,6}$ Similarly, many of the symptoms of relative adrenal insufficiency, such as hypotension and electrolyte abnormalities, are common in this population, with many possible underlying etiologies.

In the paper by Hochwald et al., ${ }^{7}$ the authors approach this dilemma by performing an ACTH stimulation test shortly after birth to obtain cortisol values in preterm infants 29 weeks and less with varying degrees of illness. They used the symptom of hypotension as a clinical marker to differentiate those infants at highest risk for relative adrenal insufficiency, and thus define an inadequate response to ACTH in the face of clinical symptoms. Using this higher risk population to establish reasonable cut points for response to ACTH provides important data specifically relevant to this unique population. Although the authors acknowledge that hypotension is multifactorial and may or may not be due to relative adrenal insufficiency, the methodology allows a narrowing of the population to symptomatic infants where the definition is most relevant. A concern has previously been raised for attempting to base a definition of relative adrenal insufficiency on cortisol values obtained shortly after birth owing to the potential for suppression of the hypothalamic-pituitary-adrenal axis from maternal steroid administration., ${ }^{8,9}$ The population presented in this study has predominantly been exposed to prenatal steroids (over $90 \%$ in both groups), allowing for a realistic evaluation comparable to the dilemma confronted by clinicians. Clearly, there is an advantage to an early diagnosis of relative adrenal insufficiency to allow appropriate clinical decision making, as shown in this study by approximately one third of the infants receiving inotropes in the first $24 \mathrm{~h}$ of life.

Other authors have used hypotension as a potential marker for relative adrenal insufficiency. $\mathrm{Ng}$ et al. ${ }^{10}$ examined ACTH stimulation tests on day 7 and day 14 of life, and found that infants with a low response to ACTH on day 7 were more likely to require inotropic therapy in the first 2 weeks of life. Basal cortisol levels alone were as predictive as the post ACTH stimulation cortisol results. Although Ng was able to document that an abnormal cortisol response was predictive of need for inotropic support, the information was clinically not timely as much of the need for inotropic therapy preceded the timing of the testing. In a multicenter trial of prophylaxis of relative adrenal insufficiency in extremely low birth weight infants, where infants were randomized to receive hydrocortisone therapy for the first 2 weeks of life, there was no difference in the need for inotropic support or the subsequent incidence of bronchopulmonary dyplasia between the treated infants compared with controls. ${ }^{11}$ When attempting to define relative adrenal insufficiency by examining cortisol values from this large cohort, low cortisol values at $24-48 \mathrm{~h}$ of life or 7-10 days of life did not predict inotropic support. Similarly, cortisol values at these two time points were not associated with bronchopulmonary dysplasia. Confounding our understanding of relative adrenal insufficiency is the difficulty of differentiating two groups of infants: those infants with inappropriately low cortisol values who may be suffering adverse effects owing to the deficient levels of the hormone, and those with developmentally appropriate low cortisol values related to prematurity and ongoing development of the hypothalamic-pituitary-adrenal axis, whose symptoms are attributable to other etiologies such as hypovolemia or sepsis.

Do we need a definition for relative adrenal insufficiency in this population of very low birth weight infants? One could argue that our treatment decisions may occur independently of having more definitive criteria for relative adrenal insufficiency. Hydrocortisone therapy is an effective adjunct treatment for pressor-resistant hypotension, and steroid therapy is well known to cause an increase in blood pressure. ${ }^{12-14}$ But hydrocortisone therapy is not without risks. Hydrocortisone therapy has been shown to be associated with gastrointestinal perforations, particularly in infants with high cortisol concentrations. ${ }^{11,15}$ There is an additional concern of drug interaction, with indomethacin use in conjunction with hydrocortisone treatment being an additional risk factor for gastrointestinal perforation. ${ }^{11}$ Thus, hydrocortisone treatment in infants who do not have relative adrenal insufficiency, especially those with high cortisol concentrations, should be considered only with great caution.

Studies have suggested an association of bronchopulmonary dysplasia and relative adrenal insufficiency. ${ }^{16,17}$ Despite this, prophylaxis of all extremely low birth weight infants did not result in a decrease in bronchopulmonary dysplasia. It remains unclear 
whether having a better definition for relative adrenal insufficiency would allow targeting of treatment to a high-risk group. Bronchopulmonary dysplasia is an end result of multiple factors promoting inflammation in a vulnerable population, ${ }^{18}$ and thus it is unclear if hydrocortisone treatment alone, beginning at what age and for what duration, is sufficient to impact the evolution of this chronic disease.

Therapies given to preterm infants should always be scrutinized for impact on long-term outcomes. To date, hydrocortisone therapy has not been associated with adverse neurodevelopmental outcomes. ${ }^{19-21}$ There is also no evidence that relative adrenal insufficiency alone is associated with adverse long termoutcomes. ${ }^{22}$ It likely that there is a subset of preterm infants with a more developmentally appropriate form of relative adrenal insufficiency, who may not require intervention or therapy that cannot be distinguished from those infants in whom relative adrenal insufficiency is a contributor to ongoing pathology. The later is the group who may benefit from appropriate treatment with hydrocortisone. Abnormal cortisol values alone, both basal and post ACTH stimulation, are not sufficient indication for requiring hydrocortisone therapy in the absence of symptoms. Further investigation is needed to continue to guide our understanding and treatment of relative adrenal insufficiency in very low birth weight infants.

\section{Conflict of interest}

The author declares no conflict of interest.

SW Aucott

Department of Pediatrics, Johns Hopkins University School of Medicine, Baltimore, MD, USA E-mail: saucott1@jbmi.edu

\section{References}

1 Cooper MS, Stewart PM. Corticosteroid insufficiency in acutely ill patients. $N$ Engl J Med 2003; 348(8): 727-734.

2 Lamberts SW, Bruining HA, de Jong FH. Corticosteroid therapy in severe illness. $N$ Engl J Med 1997; 337(18): 1285-1292.

3 Joosten KF, de Kleijn ED, Westerterp M, de Hoog M, Eijck FC, Hop WCJ et al. Endocrine and metabolic responses in children with meningoccocal sepsis: striking differences between survivors and nonsurvivors. J Clin Endocrinol Metab 2000; 85(10): $3746-3753$.
4 al Saedi S, Dean H, Dent W, Cronin C. Reference ranges for serum cortisol and 17-hydroxyprogesterone levels in preterm infants. J Pediatr 1995; 126(6): 985-987.

5 Scott SM, Watterberg KL. Effect of gestational age, postnatal age, and illness on plasma cortisol concentrations in premature infants. Pediatr Res 1995; 37(1): 112-116.

6 Huysman MW, Hokken-Koelega AC, De Ridder MA, Sauer PJ. Adrenal function in sick very preterm infants. Pediatr Res 2000; 48(5): 629-633.

7 Hochwald 0, Holsti L, Osiovich $\mathrm{H}$. The use of early ACTH test to identify hypoadrenalism-related hypotension in low birth weight infants. J Perinatol 2012; 32(6): 412-417.

8 Davis EP, Townsend EL, Gunnar MR, Georgieff MK, Guiang SF, Ciffuentes RF et al. Effects of prenatal betamethasone exposure on regulation of stress physiology in healthy premature infants. Psychoneuroendocrinology 2004; 29(8): 1028-1036.

9 Schaffer L, Luzi F, Burkhardt T, Rauh M, Beinder E. Antenatal betamethasone administration alters stress physiology in healthy neonates. Obstet Gynecol 2009; 113(5): 1082-1088.

$10 \mathrm{Ng}$ PC, Lee CH, Lam CW, Ma KC, Fok TF, Chan IH et al. Transient adrenocortical insufficiency of prematurity and systemic hypotension in very low birthweight infants. Arch Dis Child Fetal Neonatal Ed 2004; 89(2): F119-F126.

11 Watterberg KL, Gerdes JS, Cole CH, Aucott SW, Thilo EH, Mammel MC et al. Prophylaxis of early adrenal insufficiency to prevent bronchopulmonary dysplasia: a multicenter trial. Pediatrics 2004; 114(6): 1649-1657.

12 Seri I, Tan R, Evans J. Cardiovascular effects of hydrocortisone in preterm infants with pressor-resistant hypotension. Pediatrics 2001; 107(5): 1070-1074.

13 Noori S, Friedlich P, Wong P, Ebrahimi M, Siassi B, Seri I. Hemodynamic changes after low-dosage hydrocortisone administration in vasopressor-treated preterm and term neonates. Pediatrics 2006; 118(4): 1456-1466.

$14 \mathrm{Ng} \mathrm{PC}$, Lee CH, Bnur FL, Chan IH, Lee AW, Wong E et al. A double-blind, randomized, controlled study of a "stress dose" of hydrocortisone for rescue treatment of refractory hypotension in preterm infants. Pediatrics 2006; 117(2): 367-375.

15 Peltoniemi 0, Kari MA, Heinonen K, Saarela T, Nikolajev K, Andersson S et al. Pretreatment cortisol values may predict responses to hydrocortisone administration for the prevention of bronchopulmonary dysplasia in high-risk infants. J Pediatr 2005; 146(5): 632-637.

16 Watterberg KL, Gerdes JS, Cook KL. Impaired glucocorticoid synthesis in premature infants developing chronic lung disease. Pediatr Res 2001; 50(2): 190-195.

17 Coalson JJ. Pathology of bronchopulmonary dysplasia. Semin Perinatol 2006; 30(4): 179-184.

18 Jobe AH. The new bronchopulmonary dysplasia. Curr Opin Pediatr 2011; 23(2): $167-172$.

19 Watterberg KL, Shaffer ML, Mishefske MJ, Leach CL, Mammel MC, Couser RJ et al. Growth and neurodevelopmental outcomes after early low-dose hydrocortisone treatment in extremely low birth weight infants. Pediatrics 2007; 120(1): 40-48.

20 Peltoniemi OM, Lano A, Puosi R, Yliherva A, Bonsante F, Kari MA et al. Trial of early neonatal hydrocortisone: two-year follow-up. Neonatology 2009; 95(3): 240-247.

21 Rademaker KJ, de Vries LS, Uiterwaal CS, Groenendaal F, Grobbee DE, van Bel F. Postnatal hydrocortisone treatment for chronic lung disease in the preterm newborn and long-term neurodevelopmental follow-up. Arch Dis Child Fetal Neonatal Ed 2008; 93(1): F58-F63.

22 Aucott SW, Watterberg KL, Shaffer ML, Donohue PK. Early cortisol values and long-term outcomes in extremely low birth weight infants. J Perinatol 2010; 30(7): 484-488. 